\title{
EVALUATING THE EFFECTS OF SIMULATED MICROGRAVITY ON MOUSE FIBROBLAST
}

\author{
Hoang Nghia Son ${ }^{1,2}$, Hoang Nguyen Quang Huy ${ }^{1}$, Tran Thi Bich Tram ${ }^{3}$, Ly Ngoc Cang ${ }^{1}$, \\ Ho Nguyen Quynh Chi ${ }^{1,2}$, Truong Xuan Dai ${ }^{1,2}$, Le Ngoc Phuong Thanh ${ }^{1}$, Truong Thi Han ${ }^{1}$, \\ Nguyen Thai Minh Han ${ }^{1}$, Le Thanh Long ${ }^{1,2, *}$ \\ ${ }^{1}$ Animal Biotechnology Department, Institute of Tropical Biology, VAST, Vietnam \\ ${ }^{2}$ Biotechnology Department, Graduate University of Science and Technology, VAST, Vietnam \\ ${ }^{3}$ University of Science, Vietnam National University in Ho Chi Minh City
}

Received 24 March 2020, accepted 20 September 2020

\begin{abstract}
The present study investigated how mouse fibroblasts changed under microgravity (SMG) conditions $\left(<10^{-3} \mathrm{G}\right)$ simulated by $3 \mathrm{D}$ clinostat. Results showed that SMG condition markedly reduced the proliferation of mouse fibroblasts, significantly reducing the nuclear area and intensity. Compared to the control group, the mouse fibroblasts ratio of the SMG group was higher in the G0/G1 phase but lower in the S phase and G2/M phase. The ratios of early and late apoptotic cells were also higher in the SMG group. The mouse fibroblasts under SMG conditions exhibited a reduction of $\beta$-Actin and $\alpha$-Tubulin 3 expressions compared to the control group. These results suggested that the SMG condition diminished the proliferation and downregulated cytoskeletal protein expression of mouse fibroblasts.
\end{abstract}

Keywords: Cell cycle, mouse fibroblast, proliferation, simulated microgravity.

Citation: Hoang Nghia Son, Hoang Nguyen Quang Huy, Tran Thi Bich Tram, Ly Ngoc Cang, Ho Nguyen Quynh Chi, Truong Xuan Dai, Le Ngoc Phuong Thanh, Truong Thi Han, Nguyen Thai Minh Han, Le Thanh Long, 2020. Evaluating the effects of simulated microgravity on mouse fibroblast. Academia Journal of Biology, 42(3): 129-134. https://doi.org/10.15625/2615-9023/v42n3.14919

*Corresponding author email: lelongvast@gmail.com

(C2020 Vietnam Academy of Science and Technology (VAST) 


\section{INTRODUCTION}

Gravity is an omnipresent force on Earth. While influencing the movement of the whole universe, it also plays a role in the evolution of living creatures as well as in the life of all biological systems on Earth (Morey-Holton, 2003). Thus, a change of gravity in any direction can lead to significant changes in the organism (Dubinin \& Vaulina, 1976). The lack of gravity can cause disturbance in the metabolism of organs, as well as changes to bone and skin structure, etc (Ruden et al., 2018). The effects of microgravity on the human body can be clearly seen after astronauts returned from Earth. Several studies have shown that exposure to the microgravity environment will cause a range of adverse health effects for astronauts. They may suffer from bone mass loss, altered cardiovascular structure and changes in nerve organs (Carvil et al., 2013). Microgravity can affect both the nuclear shape and the cytoskeleton. The apoptosis process is also affected when the cytoskeleton is disturbed, causing apoptosis at inappropriate times (Crawford-Young, 2006).

However, it is not easy to conduct experiments in space due to high costs. Besides, while some experiments require the subject to be exposed to a microgravity environment for weeks or months, others such as the study of membrane and cytoskeleton function just need cells to be exposed to a microgravity environment for a few days. Therefore, devices that can simulate certain aspects of microgravity on the ground are indispensable and very useful for studying the impact of this environment on biological processes, gravity perception mechanisms and orientation through gravity of organisms (Clément \& Slenzka, 2006; Kim et al., 2015). Common microgravity simulation systems designed to simulate the weightlessness state or free fall in ground-based laboratories include drop tower, 2D clinostat, rotating wall vessel (RWV), random positioning or 3D clinostat (RPM). Among them, random positioning or clinostat 3D is often used to assess the effect of simulated microgravity on cells for periods lasting hours (Wuest et al., 2015).
Fibroblasts are important in creating structural proteins of ECM, binding proteins and substrates, wound healing, etc. (McAnulty, 2007; Kendall \& FeghaliBostwick, 2014). But until now, the effects of microgravity on fibroblasts have not been well studied. Therefore, this study was conducted to evaluate the effects of simulated microgravity condition on fibroblasts by evaluating fibroblast proliferation, cell cycles and cytoskeletal proteins.

\section{MATERIALS AND METHODS}

\section{Cell culture}

Mouse fibroblasts (3T3 cell line) were thawed and cultured in DMEM F-12 medium (Capricorn Scientific, Germany) supplemented with 10\% FBS (Capricorn Scientific, Germany) and $1 \%$ Penicillin/Streptomycin (Gibco, USA) at $37{ }^{\circ} \mathrm{C}, 5 \% \mathrm{CO}_{2}$. Simulated microgravity was induced using $3 \mathrm{D}$ clinostat (Migra-ITB, Institute of Tropical Biology, VAST). The clinostat was placed in a $\mathrm{CO}_{2}$ incubator and operated for three days.

\section{Cell proliferation assay}

Mouse fibroblasts were seeded to a 96well plate (Thermo Scientific, USA) with a density of $5 \times 10^{3}$ cells/well and cultured in incubator at $37{ }^{\circ} \mathrm{C}, 5 \% \mathrm{CO}_{2}$ under two gravity conditions: $1 \mathrm{G}$ condition and simulated microgravity $\left(<10^{-3} \mathrm{G}\right)$ condition. After 3 days, cell proliferation was assessed by adding $10 \mu \mathrm{l}$ WST-1 (Roche, Switzerland) to each well, and incubating for 3.5 hours at $37{ }^{\circ} \mathrm{C}, 5 \% \mathrm{CO}_{2}$. The O.D. value was measured at a wavelength of $450 \mathrm{~nm}$ using a Glomax machine (Promega, USA).

\section{Nuclear morphology evaluation}

Mouse fibroblasts were seeded to a 96well plate with a density of $5 \times 10^{3}$ cells/well and cultured in incubator at $37{ }^{\circ} \mathrm{C}, 5 \% \mathrm{CO}_{2}$ under two gravity conditions: $1 \mathrm{G}$ condition and SMG condition. After three days, cells were fixed in $4 \%$ paraformaldehyde (Nacalai Tesque, Japan) for 30 minutes, then washed twice with PBS $1 \mathrm{X}$ and treated with $0.1 \%$ Triton X100 (Sigma-Aldrich, USA) overnight at $4{ }^{\circ} \mathrm{C}$. Mouse fibroblast nuclei were stained 
with $30 \mu \mathrm{L}$ Hoechst 33342 1X (SigmaAldrich, USA) for 30 minutes and were washed twice with PBS 1X. The mouse fibroblast nucleus parameters and cell cycle progression were assessed using Cell Cycle App. of Cytell fluorescence microscope (GE Healthcare, USA).

\section{Apoptosis analysis}

Mouse fibroblasts were space to a 96-well plate with a density of $5 \times 10^{3}$ cells/well and cultured in incubator at $37{ }^{\circ} \mathrm{C}, 5 \% \mathrm{CO}_{2}$ under two gravity conditions: $1 \mathrm{G}$ condition and SMG condition. After three days, the cell cycle progression was evaluated by flow cytometry using Apoptosis Detection Kit I (BD Biosciences, United States).

\section{Western blot}

Mouse fibroblasts were collected in $1.5 \mathrm{~mL}$ tubes and treated with lysis buffer LDS 4X (Abcam, USA) at $70{ }^{\circ} \mathrm{C}$ for 10 minutes. Equal amounts of protein were loaded into wells containing SDS-PAGE gel (Abcam, USA). The gel was run for 2 hours at $50 \mathrm{~V}$. The proteins were tranferred to the PVDF membrane (Abcam, USA), which was activated in methanol (Sigma-Aldrich, USA) for three minutes. The membrane transferring was performed for 2 hours at $50 \mathrm{~V}$. Then, the membrane was blocked with blocking buffer

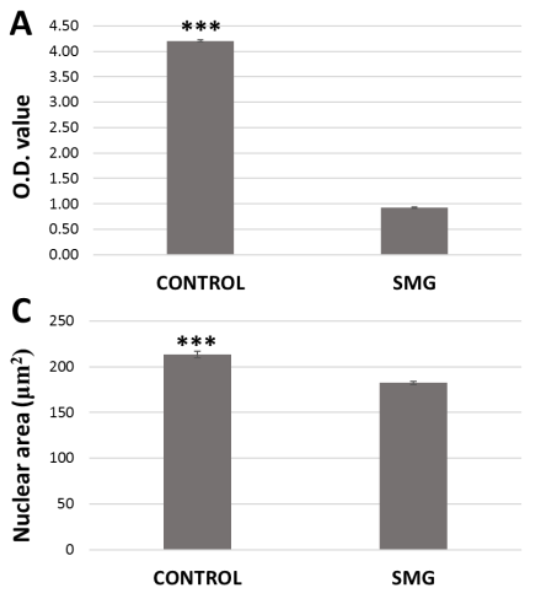

for 1 hour at room temperature (Abcam, USA), washed 3 times with TBST 1X and incubated with primary antibodies against GAPDH, $\beta$ actin, $\alpha$-tubulin 3 (Abcam, USA). The Goat anti-Rabbit secondary antibody (Abcam, USA) was used for GAPDH and $\alpha$-tubulin 3. The Goat anti-Mouse secondary antibody (Abcam, USA) was used for $\beta$-actin. The membrane was washed 3 times with TBST $1 \mathrm{X}$ and treated with a mixture of Reagent 1 and Reagent 2 in 1:1 proportion (ECL Western Blotting Substrate Kit, Abcam, USA) for 1 minute. The X-ray exposure of the membrane (Fujifilm, Japan) was carried out in a darkroom.

\section{Data analysis}

All experiments were performed at least three times. The data were analyzed using one-way ANOVA where $P<0.05$ was considered statistically significant.

\section{RESULTS AND DISCUSSION}

\section{Cell proliferation}

The WST-1 assay demonstrated that the OD value of mouse fibroblasts under SMG condition $(0.92 \pm 0.01)$ was lower than that of the control group $(4.20 \pm 0.02)(p<0.001)$ (Fig. 1A). This suggests that simulated microgravity condition markedly reduced the proliferation of mouse fibroblasts.

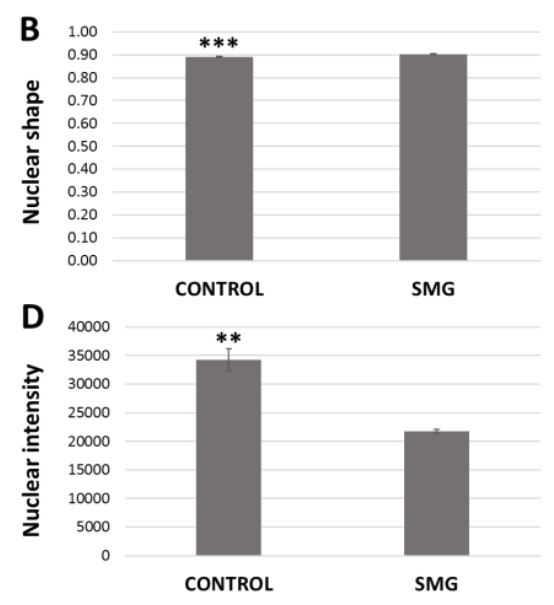

Figure 1. Changes in proliferation and nuclear of mouse fibroblast. A. Cell proliferation assessed by WST-1 assay. B. Nuclear shape value evaluation of mouse fibroblast. C. Nuclear area measurement of mouse fibroblast. D. The nuclear intensity estimation of mouse fibroblast. $* * * \mathrm{P}<0.001$ (Control group vs. SMG group), $* * \mathrm{P}<0.01$ (Control group vs. SMG group) 


\section{Cell morphology}

The nuclear shape value of mouse fibroblasts from the control group (0.89 \pm $0.003)$ was lower than that of the SMG group $(0.90 \pm 0.002)(p<0.001)$ (Fig. 1B). The mouse fibroblast nuclear areas of the control group and SMG group were $213.06 \pm 3.53 \mu \mathrm{m}^{2}$ and $182.37 \pm 1.71 \mu^{2}$, respectively (Fig. 1C). This proved that the simulated microgravity condition significantly reduced the nuclear area. The nuclear intensity of mouse fibroblasts in the SMG group (21756.54 $\pm 351.85)$ was lower than that of the control group (34252.01 \pm 1953.77) (Fig. 1D), revealing that the average nuclear intensity was significantly reduced under the effect of simulated microgravity condition.

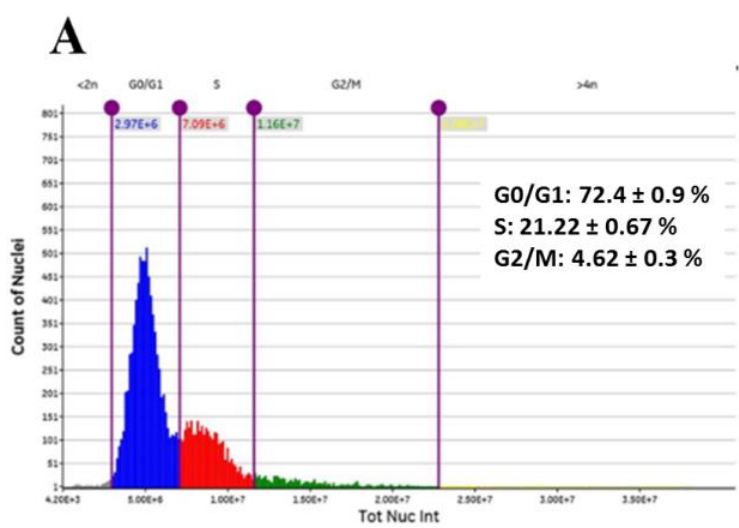

\section{Analysis of cell cycle and apoptotic cell ratios}

In the $\mathrm{G} 0 / \mathrm{G} 1$ phase, the ratio of mouse fibroblast in the SMG group is $75.88 \pm$ $0.57 \%$, higher than that of the control group $(72.4 \pm 0.9 \%)(\mathrm{p}<0.05)$ (Fig. 2). This proved that simulated microgravity condition increased the ratio of cells in the G0/G1 phase. In the $S$ phase, the ratio of mouse fibroblast in the SMG group $(17.05 \pm 0.91 \%)$ was lower than that of the control group $(21.22 \pm 0.67 \%)(p<0.05)$. Regarding mouse fibroblast percentage in $\mathrm{G} 2 / \mathrm{M}$ phase, there were no significant differences between the SMG group $(4.05 \pm 0.23 \%)$ and the control group $(4.62 \pm 0.30 \%)$.

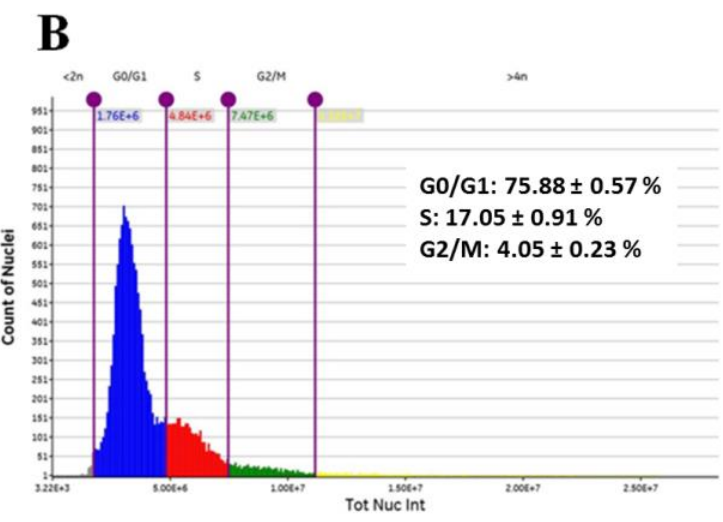

Figure 2. Cell cycle progression analysis of mouse fibroblasts from control group (A) and SMG group (B)

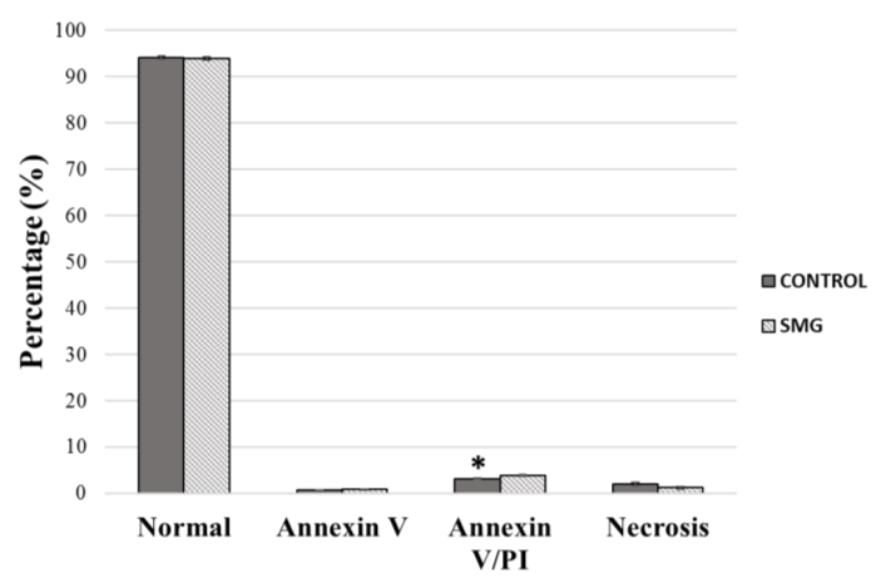

Figure 3. The apoptotic ratio of mouse fibroblast from SMG group and control group.

$$
* P<0.05 \text { (Control group vs. SMG group) }
$$


There were no significant differences in the ratio of early apoptotic cells (Annexin Vpositive) between SMG group $(0.87 \pm 0.09 \%)$ and control group $(0.67 \pm 0.09 \%)$ (Fig. 3). However, the percentage of late apoptotic cells (Annexin V-positive/PI - positive) from SMG group $(3.97 \pm 0.19 \%)$ was higher than that of the control group $(3.2 \pm 0.1 \%)$ $(\mathrm{p}<0.05)$. There were no differences in viability ratio of mouse fibroblast from the control group $(94.13 \pm 0.37 \%)$ and the SMG group $(93.97 \pm 0.35 \%)$.

\section{Expression of cytoskeletal protein}

In this study, the western blot was performed to estimate the expression of $\beta$ actin and $\alpha$-tubulin 3 proteins, which plays important role in the construction of cytoskeleton. Result showed that the expressions of $\beta$-actin and $\alpha$-tubulin 3 were downregulated in mouse fibroblast under SMG condition (Fig. 4), which indicated that SMG condition induced changes in cytoskeletal protein expression, leading to the modification of cytoskeleton.

\section{Control SMG}

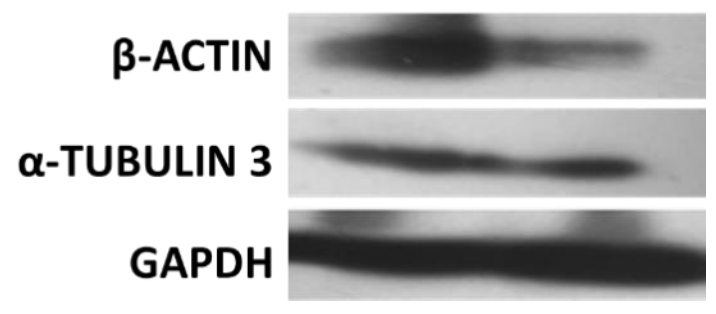

Figure 4. Western blot analysis of $\beta$-actin and $\alpha$-tubulin 3 expression in mouse fibroblasts under SMG condition and $1 \mathrm{G}$ condition (control group)

\section{Discussion}

In this study, simulated microgravity condition reduced the proliferation and nuclear area of mouse fibroblasts compared to the control group. Mouse fibroblasts being rounder in shape might be due to an increase in cell membrane actin and a decrease in central actin fibers. This showed the important role of actin in determining cell morphology as well as their sensitivity to microgravity condition (Moes et al., 2007). Cell cycle analysis showed that the ratio of mouse fibroblast from SMG group in G0/G1 phase was higher than that in the control group. This proved that the simulated microgravity condition stimulated mouse fibroblasts to enter arrest phase in the cell cycle. Moreover, the ratio of mouse fibroblasts in $S$ phase from the SMG group was lower than that of the control group. Thus, simulated microgravity conditions contributed to the reduction of DNA replication of mouse fibroblasts. In addition, there were no differences in viability ratio of mouse fibroblast between two groups.

Actin is an abundant protein present in cells in both a polymerized form (F-actin) and a non-polymerized form (G-actin) (Jiang et al., 1997). F-actin, which is formed by the polymerization of G-actin in the presence of $\mathrm{Mg}^{2+}$ and ATP, cycles between these states in cells, forming a highly dynamic network (Fletcher \& Mullins, 2010; Lodish et al., 2012). Western Blot analysis showed a decrease in actin protein expression in mouse fibroblasts from SMG group compared to the control group. Thus, this proposed that actin might be decreased by microgravity. In addition, tubulin protein also exhibited decreased expression under simulated microgravity condition.

\section{CONCLUSION}

This study demonstrated that simulated microgravity inhibited mouse fibroblast proliferation. Structural protein expression was also down-regulated, suggesting that simulated microgravity induced changes in the cytoskeletons of mouse fibroblast.

Acknowledgements: This work was supported by grant VT-CB.15/18-20 of Space Science and Technology Program from Vietnam Academy of Science and Technology.

\section{REFERENCES}

Carvil P., Baptista R., Russomano T., 2013. The human body in a microgravity environment: Long term adaptations and countermeasures. Aviat. Focus, 4(1): 10-22. 
Clément G., Slenzka K., 2006. Fundamentals of Space Biology: Research on Cells, Animals, and Plants in Space. SpringerVerlag New York Inc, New York, USA.

Crawford-Young S. J., 2006. Effects of microgravity on cell cytoskeleton and embryogenesis. Int. J. Dev. Biol., 50(2-3): 183-91.

Dubinin N. P., Vaulina E. N., 1976. The evolutionary role of gravity. Life Sci. Space Res., 14: 47-55.

Fletcher D., Mullins R., 2010. Cell mechanics and the cytoskeleton. Nature, 463: 485-492.

Jiang C. J., Weeds A. G., Khan S., Hussey P. J. 1997. F-actin and G-actin binding are uncoupled by mutation of conserved tyrosine residues in maize actin depolymerizing factor (ZmADF). Proceedings of the National Academy of Sciences, 94(18): 9973-9978.

Kendall R. T., Feghali-Bostwick C. A., 2014. Fibroblasts in fibrosis: novel roles and mediators. Front. Pharmacol., 5: 123.

Kim Y. K., Park S. H., Lee J. H., Choi G. H., 2015. Design and Performance of an Automated Bioreactor for Cell Culture Experiments in a Microgravity Environment. J. Astron. Space Sci., 2(1): 81-89.

Lodish H., Kaiser C. A., Krieger M., Bretscher A., Ploegh H., Amon A., Scott
M. P., 2012. Molecular Cell Biology. W.H. Freeman, Dallas, TX, USA chapter 4: 181-265.

McAnulty R. J., 2007. Fibroblasts and myofibroblasts: Their source, function and role in disease. Int. J. Biochem. Cell Biol., 39(4): 666-671.

Moes M. J. A., Bijvelt J. J., Boonstra J., 2007. Actin dynamics in mouse fibroblasts in microgravity. Microgravity Sci. Technol., 19: $180-183$.

Morey-Holton E. R., 2003. The impact of gravity on life. In: Rothschild LJ, Lister AM (eds) Evolution on Planet Earth: The Impact of the Physical Environment, Academic Press, Cambridge, Massachusetts, USA pp. 143-146.

Ruden D. M., Bolnick A., Awonuga A., Abdulhasan M., Perez G., Puscheck E. E., Rappolee D. A., 2018. Effects of Gravity, Microgravity or Microgravity Simulation on Early Mammalian Development. Stem Cells Dev., 27(18): 1230-1236.

Sherr C. J., Beach D., Shapiro G. I., 2016. Targeting Cdk4 and Cdk6: From discovery to therapy. Cancer Discov., 6(4): 353-367.Wuest S. L., Richard S., Kopp S., Grimm D., Egli M., 2015. Simulated Microgravity: Critical Review on the Use of Random Positioning Machines for Mammalian Cell Culture. Biomed Res. Int., Article ID: 971474: 1-8. 